\title{
Recent advances in voxel-based targeted radionuclide therapy dosimetry
}

\author{
Greta S. P. Mok ${ }^{1,2}$, Yuni K. Dewaraja ${ }^{3}$ \\ ${ }^{1}$ Biomedical Imaging Laboratory (BIG), Department of Electrical and Computer Engineering, Faculty of Science and Technology, University \\ of Macau, Macau, China; ${ }^{2}$ Center for Cognitive and Brain Sciences, Institute of Collaborative Innovation, University of Macau, Macau, China; \\ ${ }^{3}$ Department of Radiology, University of Michigan Medical School, Ann Arbor, MI, USA
}

Correspondence to: Greta S. P. Mok, PhD. Department of Electrical and Computer Engineering, Faculty of Science and Technology, University of Macau, Taipa, Macau, China. Email: gretamok@um.edu.mo.

Submitted Sep 03, 2020. Accepted for publication Sep 27, 2020.

doi: 10.21037/qims-20-1006

View this article at: http://dx.doi.org/10.21037/qims-20-1006

Targeted radionuclide therapy (TRT) is recognized as an effective means for treating a variety of cancers (1), ranging from conventional ${ }^{131} \mathrm{I}$-radioiodine for differential thyroid carcinoma (DTC), ${ }^{223} \mathrm{Ra}$-Dichloride for bone metastasis of castration-resistant prostate cancer (CRPC), ${ }^{90} \mathrm{Y}$ microspheres for hepatic cancers, to newly EMA (Europe) and FDA (USA) approved ${ }^{177} \mathrm{Lu}$-DOTATATE Peptide Receptor Radionuclide Therapy (PRRT) for neuroendocrine tumors. Furthermore, several other therapeutic agents including ${ }^{177} \mathrm{Lu}$-PSMA for prostate cancers and alpha-particle emitters for treating different cancers are in clinical trial or are being developed and evaluated (2). Personalized treatment planning can ensure TRT efficacy while avoiding potential toxicity to critical organs by considering patient-specific pharmacokinetics. Sequential radionuclide imaging following a pre-therapy tracer administration can serve as a non-invasive tool for predicting the radiation absorbed doses delivered to tumor and critical organs by the therapy. In the case of therapies administered over multiple cycles, such as ${ }^{177} \mathrm{Lu}-$ DOTATATE, imaging-based dosimetry after one cycle can be used to predict the absorbed doses that will be delivered by subsequent cycles for consideration of potential dosage adjustment. Quantitative emission computed tomography (ECT), i.e., single photon emission computed tomography (SPECT) and positron emission tomography (PET), provides 3-dimensional (3D) activity distributions for voxel-level dosimetry, which is of increasing research and commercial interest in TRT $(3,4)$. Sequential ECT images can be directly converted to dose-rate maps or a time integrated activity (TIA) map, which can then be converted to an absorbed dose map.

Conventional corrections for diagnostic agents in ECT include uniformity, scatter, attenuation, detector response and sometimes partial volume effect. Depth dependent collimator-detector response correction is important for SPECT as well as correction of random coincidences for PET. For therapeutic radionuclides used in TRT, besides the conventional corrections, special attention should be placed on down scatter and collimator penetration for SPECT (5); multi-energy window selection and scatter correction for Bremsstrahlung imaging; prompt gamma emission and increased positron range for PET as their decay schemes are usually complex and associated with high energy positrons/photons emissions, even for their imaging surrogates. Partial volume correction is also important for all imaging modalities in TRT generally. Instead of the organ/lesion-level partial volume correction using recovery coefficients, the need for voxellevel correction has been recognized and solutions are being investigated $(6,7)$. With appropriate modelling, quantitative accuracy can reach $>90 \%$ for PET (8), SPECT (9) and Bremsstrahlung imaging (10). Clinically, state-of-the-art spatial resolutions of 6-8 $\mathrm{mm}$ (11) or even $<3 \mathrm{~mm}$ (12) for dedicated organs in SPECT, 4-6 mm in PET (13) have been reported. Considering the travelling 
distance for common beta $(1.5-19 \mathrm{~mm})$ and alpha particles $(0.016-0.075 \mathrm{~mm})(14)$ associated with TRT agents, the spatial resolution and thus resolvable voxel size would need to be further improved to reduce the uncertainties in dose estimation at the voxel-level $(6,15)$. Techniques such as time-of-flight PET, point spread function modelling, solidstate detectors, digital ECT using silicon photomultiplier tubes with photon counting capability have demonstrated improved quantitative imaging performance and hold promise for improving TRT voxel-based dosimetry. Novel collimators designs are possible ways to improve SPECT and Bremsstrahlung imaging (16). For example, pinhole collimators are shown to reduce collimator penetration for I-123 imaging (17). However, factors such as field-ofview and sensitivity would need to be considered when designing collimators as acquisition time is important for patient throughput in clinical TRT practice. Besides, respiratory motion degrades image quality particularly near the lung-liver interface. Therefore, it may impact the quantitative accuracy and dosimetric estimation for post-therapy ${ }^{90} \mathrm{Y} \mathrm{PET} /$ Bremsstrahlung imaging and pretherapy ${ }^{99 \mathrm{~m}}$ Tc-macro aggregated albumin (MAA) SPECT for treatment planning of microsphere radioembolization in hepatic cancers. Respiratory gating via external tracking devices $(18,19)$ as well as fully data-driven approaches $(20)$ can be used to alleviate this problem in SPECT (21) and PET (22). New initiatives for imaging instrumentation dedicated for TRT are underway (23).

Usually patients are imaged at multiple time points for TRT dosimetry to obtain the time activity curves (TAC) for critical organs and tumors. Accurate alignment between these sequential images is of much importance for $3 \mathrm{D}$ dosimetry (24). Multiple research groups have performed voxel-level spatial alignment for dosimetry $(25,26)$. Our group at University of Macau has demonstrated that voxelbased non-rigid registration from sequential SPECT is feasible to enhance the 3D absorbed dose estimations and cumulative dose volume histograms (27). The integrated CT or MRI images can be used to further improve the precision of sequential ECT image registration and segmentation (28), while registration between ECT and CT/MRI at the same time point is also important to avoid errors from misaligned attenuation maps and volumesof-interest defined on the anatomical image that are applied to ECT images (29). Repeated CT scans paired with the corresponding sequential ECTs are nevertheless not in routine practice due to the concern of increased radiation exposure and a single CT is usually acquired. Some groups rely on SPECT/CT at a single time point coupled with planar whole body imaging to estimate pharmacokinetics (30). If multiple SPECT/CTs are performed, the CT exposure can be minimized by reducing the tube current substantially at all but one imaging time points, or by the "virtual CT" method proposed by our group (31). On the other hand, ${ }^{90} \mathrm{Y}$ microsphere imaging is a relatively simplified TRT application as only 1 imaging time point is generally needed. The microspheres are assumed to be trapped in the microcapillaries with only physical decay afterwards, thus multi-time point imaging and registration are not performed. Furthermore, because ${ }^{90} \mathrm{Y}$ is an almost pure beta emitter, reasonably accurate voxelbased dosimetry can be performed assuming local energy deposition without the need to consider photon-transport. However, imaging of ${ }^{90} \mathrm{Y}$ is complex as it relies on SPECT imaging of Bremsstrahlung photons and PET imaging of annihilated photons from a very low positron branching ratio. Specialized reconstruction methods including methods based on Monte Carlo simulations $(10,32,33)$ and neural networks $(34,35)$ have been proposed to address the challenges of ${ }^{90} \mathrm{Y}$ imaging. The use of PET/MR could be beneficial for the radioembolization application particularly due to the superior soft tissue contrast provided by MR for tumor segmentation in the liver (36). Automatic and reliable segmentation methods would be desirable to alleviate the processing time and inter/intra operator inconsistency (37) especially for applications with many organs of interest and multiple imaging sessions.

Curve fitting is usually used to obtain the TACs from multiple time point imaging data and is generally considered to be more accurate than numerical integration for estimating TIA. Mono- or multi-exponential models are typically employed depending on number of imaging time points and the tracer pharmacokinetics, while best fit can be obtained from certain criteria testing and minimizing the errors as compared to the measurements $(38,39)$. Recently proposed approaches for approximating TIA based on single time point ${ }^{177} \mathrm{Lu}$-PSMA (40), ${ }^{177} \mathrm{Lu}$-DOTATATE (41) and ${ }^{90}$ Y-DOTATOC (42) imaging data would greatly enhance the clinical feasibility of personalized dosimetry considering limited reimbursement, patient compliance and clinical resources. However, selection of an optimal imaging time point for different 
organs of interest, tumors and tracers is critical and this method may not be suitable for outlier patients with unique pharmacokinetics.

The gold standard for converting TIA maps to voxellevel absorbed dose maps (or activity maps to doserate maps) is based on Monte Carlo simulations (MCS) (43-45) with inputs of TIA (or activity) maps and CTbased density maps. The long computational time impedes its implementation in the clinic. An efficient alternate is to use dose point kernel (DPK)/voxel-S-value (VSV) method for dose conversion. This method is based on kernels of different voxel sizes, isotopes and media generated by MCS while many of them are tabulated in the literature (46). Its accuracy is shown to be comparable to full MCS in soft tissues generally, but substantial deviations have been shown at the lung-liver interface for ${ }^{90} \mathrm{Y}$ microspheres (47) or lungs for ${ }^{177} \mathrm{Lu}$ applications (48), even when tissuesspecific kernels were assigned (49). New dose kernels to address the tissue heterogeneity problem are being developed (50). Lately, deep learning methods have been proposed to generate $3 \mathrm{D}$ absorbed dose maps or dose rate maps directly from TIA or activity maps with high accuracy and speed, using convolutional neural network (CNN) $(51,52)$ or deep neural network (DNN) (53). Although the training process based on ECT/CT images paired with their MCS generated absorbed dose maps for specific TRT agents can be time consuming, this process is performed only one time in general. Prior to clinical use of these new methods, sufficient data are required for the training/testing process to establish generalizability.

There are other exciting ongoing developments in TRT dosimetry. While dosimetry is currently mostly performed on beta and gamma emitters, alpha particles hold promise for TRT due to their high linear energy transfer (LET) for killing cancer cells in a short range while sparing normal tissues. Microdosimetry is more suitable for alpha particles and is being investigated by several groups $(54,55)$, yet its standard imaging protocols are to be established (56). Besides, as precise voxel-based dosimetry would require a dedicated physicist or research personnel which may not be available in many clinics, a robust one-stop internal dosimetry software package for image registration, segmentation, curve fitting, dose conversion and 3D dose analysis such as isodose contours and dose volume histograms would be paramount for practical clinical implementation of TRT dosimetry. Various research
(57-63) and commercial (64-66) internal dosimetry software is now available, with enhanced functions being developed. Figure 1 shows an example of voxel-level dosimetry based on multiple SPECT/CT imaging sessions for a patient undergoing ${ }^{177} \mathrm{Lu}$-DOTATATE PRRT using the Dose Planning Method (DPM) Monte Carlo software (67). Some programs $(62,63)$ are integrated with models to estimate radiobiologic dose-metrics such as biological effective dose (BED) and equivalent uniform dose (EUD), although the values of model parameters, such as lesion/normal-tissue radiosensitivity, are not yet well established. Besides, as voxel-based calculation for aforementioned dosimetric operations, e.g., registration, curve fitting and segmentation can be computational demanding, deep learning or GPU-based computation can be feasible options to accelerate the voxel-based dose estimation process. After all, development and testing of new dosimetric methods require access to clinical patient images. Currently, University of Michigan is establishing a data-sharing repository of anonymized patient PET/CT and SPECT/ CT imaging datasets for various therapeutic agents (68). Access to such data is expected to facilitate research in TRT dosimetry and multi-institutional collaborations among researchers or even clinicians since post-therapy imaging data are still not typically acquired at most clinics.

Albeit there have been ongoing debates regarding the necessities of personalized (69) and voxel-based dosimetry (70), most agree that one-dose-fits-all is not the best way to treat patients as dose escalation or reduction is necessary for efficacious and safe treatment of some patients. Voxel-based method provides more accurate and complete dosimetric information such as heterogeneous absorbed dose distribution within the same organ as compared to the conventional mean absorbed dose method. This information is particularly useful for organs with substructures such as cortex and medulla in kidneys. With standardized dosimetric methodologies, reliable software, advance imaging technologies more available to reduce the dosimetric uncertainties and demand on clinical resources, voxel-based dosimetry is becoming feasible for routine clinical practice in various TRT applications. More systematic studies are warranted to demonstrate the absorbed dose-effect relationships and potential improvement in clinical outcome for "confident" personalized voxel-based dosimetry. 


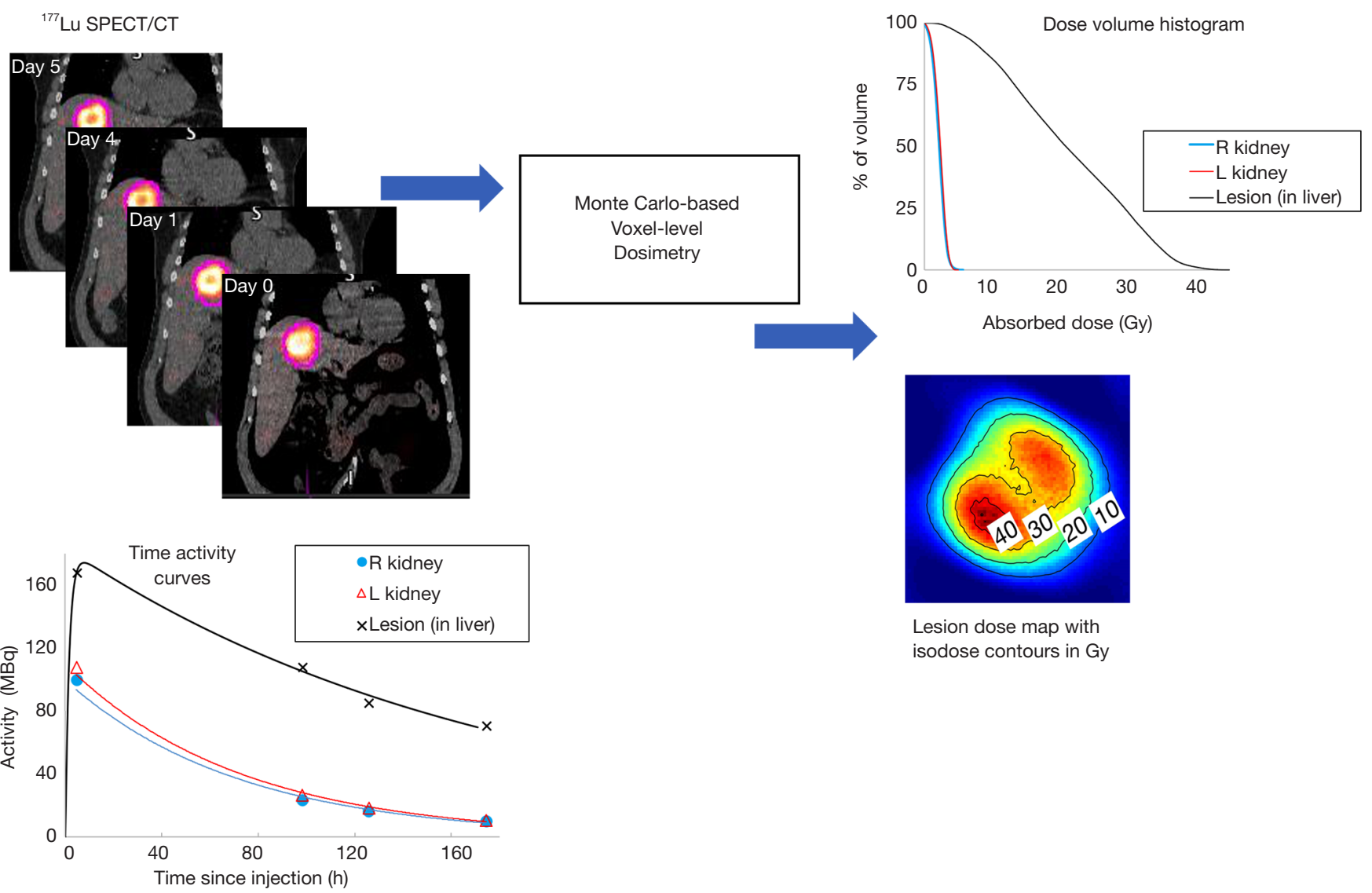

Figure 1 Sample dose volume histograms (DVHs) and lesion absorbed dose map corresponding to a patient imaged at 4 time points after cycle 1 of standard (7.4 GBq) 177Lu DOTATATE PRRT. SPECT/CT images at each time point were input to a Monte Carlo dosimetry code and the corresponding dose-rate maps were integrated to derive the absorbed dose map (67). Mean absorbed doses were: lesion 22 Gy, R kidney 2.5 Gy, L kidney 2.6 Gy.

\section{Acknowledgments}

Funding: The authors would like to acknowledge research funding from University of Macau (MYRG2017-00060FST) and the National Institutes of Health, USA (R01CA240706 and R01EB022075). The content is solely the responsibility of the authors and does not necessarily represent the official views of the National Institutes.

\section{Footnote}

Provenance and Peer Review: This article was commissioned by the editorial office, Quantitative Imaging in Medicine and Surgery. The article did not undergo external peer review.

Conflicts of Interest: Both authors have completed the ICMJE uniform disclosure form (available at http://dx.doi. org/10.21037/qims-20-1006). Dr. GSPM serves as an unpaid editorial board member of Quantitative Imaging in Medicine and Surgery; she reports that she has a China software copyright registration for an internal dosimetry software (BIGDOSE). Dr. YKD reports personal fees from MIM Software, Inc., USA, outside the submitted work.

Open Access Statement: This is an Open Access article distributed in accordance with the Creative Commons Attribution-NonCommercial-NoDerivs 4.0 International License (CC BY-NC-ND 4.0), which permits the noncommercial replication and distribution of the article with the strict proviso that no changes or edits are made and the original work is properly cited (including links to both the formal publication through the relevant DOI and the license). See: https://creativecommons.org/licenses/by-nc-nd/4.0/. 


\section{References}

1. Goldsmith SJ. Targeted radionuclide therapy: a historical and personal review. Semin Nucl Med 2020;50:87-97.

2. Langbein T, Weber WA, Eiber M. Future of theranostics: an outlook on precision oncology in nuclear medicine. J Nucl Med 2019;60:13S-9S.

3. Li T, Ao EC, Lambert B, Brans B, Vandenberghe S, Mok GS. Quantitative imaging for targeted radionuclide therapy dosimetry-technical review. Theranostics 2017;7:4551.

4. Ljungberg M, Gleisner KS. 3-D Image-Based Dosimetry in Radionuclide Therapy. IEEE Trans Radiat Plasma Med Sci 2018;2:527-40.

5. Dewaraja YK, Frey EC, Sgouros G, Brill AB, Roberson P, Zanzonico PB, Ljungberg M. MIRD pamphlet no. 23: quantitative SPECT for patient-specific 3-dimensional dosimetry in internal radionuclide therapy. J Nucl Med 2012;53:1310-25.

6. Tran-Gia J, Salas-Ramirez M, Lassmann M. What You See Is Not What You Get: On the Accuracy of VoxelBased Dosimetry in Molecular Radiotherapy. J Nucl Med 2020;61:1178-86.

7. Thomas BA, Cuplov V, Bousse A, Mendes A, Thielemans K, Hutton BF, Erlandsson K. PETPVC: a toolbox for performing partial volume correction techniques in positron emission tomography. Phys Med Biol 2016;61:7975-93.

8. El-Ali HH, Eckerwall M, Skovgaard D, Larsson E, Strand $\mathrm{S}-\mathrm{E}, \mathrm{Kjaer}$ A. The Combination of In vivo 124I-PET and CT small animal imaging for evaluation of thyroid physiology and dosimetry. Diagnostics 2012;2:10-22.

9. He B, Du Y, Song X, Segars WP, Frey EC. A Monte Carlo and physical phantom evaluation of quantitative In-111 SPECT. Phys Med Biol 2005;50:4169-85.

10. Rong X, Du Y, Ljungberg M, Rault E, Vandenberghe S, Frey EC. Development and evaluation of an improved quantitative 90Y bremsstrahlung SPECT method. Med Phys 2012;39:2346-58.

11. Ljungberg M, Pretorius PH. SPECT/CT: an update on technological developments and clinical applications. Br J Radiol 2018;91:20160402.

12. Chen Y, Vastenhouw B, Wu C, Goorden MC, Beekman FJ. Optimized image acquisition for dopamine transporter imaging with ultra-high resolution clinical pinhole SPECT. Phys Med Biol 2018;63:225002.

13. Zhang J, Maniawski P, Knopp MV. Performance evaluation of the next generation solid-state digital photon counting
PET/CT system. EJNMMI Res 2018;8:97.

14. Wilbur DS. The Radiopharmaceutical Chemistry of Alpha-Emitting Radionuclides. In: Lewis J, Windhorst A, Zeglis B, editors. Radiopharmaceutical Chemistry. Cham: Springer, 2019.

15. Gear JI, Cox MG, Gustafsson J, Gleisner KS, Murray I, Glatting G, Konijnenberg M, Flux GD. EANM practical guidance on uncertainty analysis for molecular radiotherapy absorbed dose calculations. Eur J Nucl Med Mol Imaging 2018;45:2456-74.

16. Rong X, Frey EC. A collimator optimization method for quantitative imaging: Application to Y-90 bremsstrahlung SPECT. Med Phys 2013;40:082504.

17. Könik A, Auer B, De Beenhouwer J, Kalluri K, Zeraatkar N, Furenlid LR, King MA. Primary, scatter, and penetration characterizations of parallel-hole and pinhole collimators for I-123 SPECT. Phys Med Biol 2019;64:245001.

18. Zhang D, Pretorius PH, Ghaly M, Zhang Q, King MA, Mok GSP. Evaluation of different respiratory gating schemes for cardiac SPECT. J Nucl Cardiol 2020;27:634-47.

19. Zhang D, Sun J, Pretorius PH, King M, Mok GS. Clinical evaluation of three respiratory gating schemes for different respiratory patterns on cardiac SPECT. Med Phys 2020;47:4223-32.

20. Kesner AL, Schleyer PJ, Büther F, Walter MA, Schäfers KP, Koo PJ. On transcending the impasse of respiratory motion correction applications in routine clinical imaging-a consideration of a fully automated data driven motion control framework. EJNMMI Phys 2014;1:8.

21. Bastiaannet R, Viergever MA, de Jong HWA. Impact of respiratory motion and acquisition settings on SPECT liver dosimetry for radioembolization. Med Phys 2017;44:5270-9.

22. Osborne DR, Acuff SN, Neveu ML, Syed M, Kaman $\mathrm{AD}, \mathrm{Fu}$ Y. Feasibility assessment of yttrium-90 liver radioembolization imaging using amplitude-based gated PET/CT. Nucl Med Commun 2018;39:222.

23. Engineering New Instrumentation for Imaging Unsealed Source Radiotherapy Agents, Virtual Meeting, National Institutes of Health, USA. 2020. Available online: https:// www.imagingtherapy.nibib.nih.gov/

24. Papavasileiou P, Divoli A, Hatziioannou K, Flux GD. The importance of the accuracy of image registration of SPECT images for 3D targeted radionuclide therapy dosimetry. Phys Med Biol 2007;52:N539-48.

25. Jackson PA. Contour-guided Registration. Available online: https://github.com/jacksonmedphysics/contour- 
guided-registration

26. Papavasileiou P, Divoli A, Hatziioannou K, Flux GD. A generalized 4D image registration scheme for targeted radionuclide therapy dosimetry. Cancer Biother Radiopharm 2007;22:160-5.

27. Ao EC, Wu NY, Wang SJ, Song N, Mok GS. Improved dosimetry for targeted radionuclide therapy using nonrigid registration on sequential SPECT images. Med Phys 2015;42:1060-70.

28. Li T, Wu NY, Song N, Mok GS. Evaluation of sequential SPECT and CT for targeted radionuclide therapy dosimetry. Ann Nucl Med 2018;32:34-43.

29. He B, Frey EC. The impact of $3 \mathrm{D}$ volume of interest definition on accuracy and precision of activity estimation in quantitative SPECT and planar processing methods. Phys Med Biol 2010;55:3535-44.

30. Sundlöv A, Sjögreen-Gleisner K, Svensson J, Ljungberg M, Olsson T, Bernhardt P, Tennvall J. Individualised 177LuDOTATATE treatment of neuroendocrine tumours based on kidney dosimetry. Eur J Nucl Med Mol Imaging 2017;44:1480-9.

31. Li T, Mok GS. Virtual CT for reducing CT dose in targeted radionuclide therapy dosimetry. Med Phys 2018;45:5138-44.

32. Dewaraja YK, Chun SY, Srinivasa RN, Kaza RK, Cuneo KC, Majdalany BS, Novelli PM, Ljungberg M, Fessler JA. Improved quantitative 90Y bremsstrahlung SPECT/CT reconstruction with Monte Carlo scatter modeling. Med Phys 2017;44:6364-76.

33. Elschot M, Lam MG, van den Bosch MA, Viergever MA, de Jong HW. Quantitative Monte Carlo-based 90Y SPECT reconstruction. J Nucl Med 2013;54:1557-63.

34. Xiang H, Lim H, Fessler JA, Dewaraja YK. A deep neural network for fast and accurate scatter estimation in quantitative SPECT/CT under challenging scatter conditions. Eur J Nucl Med Mol Imaging 2020;47:2956-67.

35. Lim H, Chun IY, Dewaraja YK, Fessler JA. Improved low-count quantitative PET reconstruction with an iterative neural network. IEEE Trans Med Imaging 2020;39:3512-22.

36. Knešaurek K, Tuli A, Kim E, Heiba S, Kostakoglu L. Comparison of PET/CT and PET/MR imaging and dosimetry of yttrium-90 (90 Y) in patients with unresectable hepatic tumors who have received intraarterial radioembolization therapy with $90 \mathrm{Y}$ microspheres. EJNMMI Phys 2018;5:23.

37. Lamba N, Wan H, Kruzer A, Platt E, Nelson A. Clinical utility of a 3D convolutional neural network kidney segmentation method for radionuclide dosimetry. J Nucl Med 2019;60:S267.

38. Glatting G, Kletting P, Reske SN, Hohl K, Ring C. Choosing the optimal fit function: Comparison of the Akaike information criterion and the F-test. Med Phys 2007;34:4285-92.

39. Sarrut D, Halty A, Badel JN, Ferrer L, Bardiès M. Voxel-based multimodel fitting method for modeling time activity curves in SPECT images. Med Phys 2017;44:6280-8.

40. Jackson PA, Hofman MS, Hicks RJ, Scalzo M, Violet J. Radiation Dosimetry in 177Lu-PSMA-617 Therapy Using a Single Posttreatment SPECT/CT Scan: A Novel Methodology to Generate Time-and Tissue-Specific Dose Factors. J Nucl Med 2020;61:1030-6.

41. Hänscheid H, Lapa C, Buck AK, Lassmann M, Werner RA. Dose mapping after endoradiotherapy with $177 \mathrm{Lu}-$ DOTATATE/DOTATOC by a single measurement after 4 days. J Nucl Med 2018;59:75-81.

42. Madsen MT, Menda Y, O'Dorisio TM, O'Dorisio MS. Single time point dose estimate for exponential clearance. Med Phys 2018;45:2318-24.

43. Jan S, Santin G, Strul D, Staelens S, Assie K, Autret D, Avner S, Barbier R, Bardies M, Bloomfield P. GATE: a simulation toolkit for PET and SPECT. Phys Med Biol 2004;49:4543.

44. Yoriyaz H, Stabin MG, dos Santos A. Monte Carlo MCNP-4B-based absorbed dose distribution estimates for patient-specific dosimetry. J Nucl Med 2001;42:662-9.

45. Wilderman SJ, Dewaraja YK. Method for Fast CT/ SPECT-Based 3D Monte Carlo Absorbed Dose Computations in Internal Emitter Therapy. IEEE Trans Nucl Sci 2007;54:146-51.

46. Graves SA, Flynn RT, Hyer DE. Dose point kernels for 2,174 radionuclides. Med Phys 2019;46:5284-93.

47. Mikell JK, Mahvash A, Siman W, Mourtada F, Kappadath SC. Comparing voxel-based absorbed dosimetry methods in tumors, liver, lung, and at the liver-lung interface for $90 \mathrm{Y}$ microsphere selective internal radiation therapy. EJNMMI Phys 2015;2:16.

48. Götz T, Schmidkonz C, Lang EW, Maier A, Kuwert T, Ritt P. A comparison of methods for adapting dosevoxel-kernels to tissue inhomogeneities. Phys Med Biol 2019;64:245011.

49. Khazaee Moghadam M, Kamali Asl A, Geramifar P, Zaidi $H$. Evaluating the application of tissue-specific dose kernels instead of water dose kernels in internal dosimetry: a Monte Carlo study. Cancer Biother Radiopharm 
2016;31:367-79.

50. Graves S, Tiwari A, Sunderland J. Collapsed-cone convolution superposition for improved accuracy of voxelwise dosimetry. J Nucl Med 2020;61:S535.

51. Lee MS, Hwang D, Kim JH, Lee JS. Deep-dose: a voxel dose estimation method using deep convolutional neural network for personalized internal dosimetry. Sci Rep 2019;9:10308.

52. Li Z, Fessler JA, Mikell J, Wilderman SJ, Dewaraja YK. A Deep Residual Learning Network for Practical Voxel Dosimetry in Radionuclide Therapy. IEEE Nuclear Science Symposium and Medical Imaging Conference (NSS-MIC); Boston, MA 2020. Available online: https:// www.eventclass.org/contxt_ieee2020/online-program/ session?s=M-17

53. Götz TI, Schmidkonz C, Chen S, Al-Baddai S, Kuwert T, Lang E. A deep learning approach to radiation dose estimation. Phys Med Biol 2020;65:035007.

54. Hofmann W, Li WB, Friedland W, Miller BW, Madas $\mathrm{B}$, Bardiès M, Balásházy I. Internal microdosimetry of alpha-emitting radionuclides. Radiat Environ Biophys 2020;59:29-62.

55. Sgouros G, Hobbs R, Josefsson A. Dosimetry and radiobiology of alpha-particle emitting radionuclides. Curr Radiopharm 2018;11:209-14.

56. Benabdallah N, Scheve W, Dunn N, Silvestros D, Schelker P, Abou D, Laforest R, Dehdashti F, Thorek D. Practical Considerations for Quantitative Clinical SPECT/CT Imaging of Alpha Particle Emitting Radioisotopes. J Nucl Med 2020;61:S532.

57. Li T, Zhu L, Lu Z, Song N, Lin KH, Mok GS. BIGDOSE: software for 3D personalized targeted radionuclide therapy dosimetry. Quant Imaging Med Surg 2020;10:160.

58. Jackson PA, Beauregard JM, Hofman MS, Kron T, Hogg A, Hicks RJ. An automated voxelized dosimetry tool for radionuclide therapy based on serial quantitative SPECT/ CT imaging. Med Phys 2013;40:112503.

59. Grimes J, Uribe C, Celler A. JADA: a graphical user interface for comprehensive internal dose assessment in nuclear medicine. Med Phys 2013;40:072501.

60. Kost SD, Dewaraja YK, Abramson RG, Stabin MG. VIDA: a voxel-based dosimetry method for targeted radionuclide therapy using Geant4. Cancer Biother Radiopharm 2015;30:16-26.

61. Kletting P, Schimmel S, Hänscheid H, Luster M, Fernández M, Nosske D, Lassmann M, Glatting G. The NUKDOS software for treatment planning in molecular radiotherapy. Zeitschrift für Medizinische Physik
2015;25:264-74.

62. Dewaraja YK, Schipper MJ, Roberson PL, Wilderman SJ, Amro H, Regan DD, Koral KF, Kaminski MS, Avram AM. 131I-tositumomab radioimmunotherapy: Initial tumor doseresponse results using 3-dimensional dosimetry including radiobiologic modeling. J Nucl Med 2010;51:1155-62.

63. Sgouros G, Hobbs RF, Atkins FB, Van Nostrand D, Ladenson PW, Wahl RL. Three-dimensional radiobiological dosimetry (3D-RD) with 124 I PET for 131 I therapy of thyroid cancer. Eur J Nucl Med Mol Imaging 2011;38:S41-7.

64. Grassi E, Fioroni F, Ferri V, Mezzenga E, Sarti M, Paulus T, Lanconelli N, Filice A, Versari A, Iori M. Quantitative comparison between the commercial software STRATOS® by Philips and a homemade software for voxel-dosimetry in radiopeptide therapy. Phys Med 2015;31:72-9.

65. Mora-Ramirez E, Santoro L, Cassol E, Ocampo-Ramos JC, Clayton N, Kayal G, Chouaf S, Trauchessec D, Pouget JP, Kotzki PO, Deshayes E, Bardiès M. Comparison of commercial dosimetric software platforms in patients treated with $177 \mathrm{Lu}$-DOTATATE for peptide receptor radionuclide therapy. Med Phys 2020;47:4602-15.

66. Hippeläinen ET, Tenhunen MJ, Mäenpää HO, Heikkonen JJ, Sohlberg AO. Dosimetry software Hermes Internal Radiation Dosimetry: from quantitative image reconstruction to voxel-level absorbed dose distribution. Nucl Med Commun 2017;38:357-65.

67. Dewaraja Y, Wilderman S, Niedbala J, Frey K, Wong KK. Multi SPECT/CT-based patient specific lesion and kidney dosimetry for verification of simpler approaches for treatment planning in Lu-177 DOTATATE PRRT. J Nucl Med 2019;60:S1626.

68. University of Michigan Deep Blue Data. Available online: https://deepblue.lib.umich.edu/data.

69. Stabin MG, Madsen MT, Zaidi H. Personalized dosimetry is a must for appropriate molecular radiotherapy. Med Phys 2019;46:4713-6.

70. Chiesa C, Bardiès M, Zaidi H. Voxel-based dosimetry is superior to mean absorbed dose approach for establishing dose-effect relationship in targeted radionuclide therapy. Med Phys 2019;46:5403-6.

Cite this article as: Mok GSP, Dewaraja YK. Recent advances in voxel-based targeted radionuclide therapy dosimetry. Quant Imaging Med Surg 2021;11(2):483-489. doi: 10.21037/qims-201006 